Ilchmann, Achim; Pinho, Maria do Rosário de :

Weak maximum principle for optimal control problems with mixed constraints

Zuerst erschienen in:

Nonlinear Analysis 48 (2002), Nr. 8, S. 1179-1196

DOI: $10.1016 / \mathrm{S} 0362-546 \mathrm{X}(01) 00094-3$ 


\title{
Weak maximum principle for optimal control problems with mixed constraints
}

\author{
Maria do Rosário de Pinho* and Achim Ilchmann ${ }^{\dagger}$ \\ * Departamento de Engenharia Electrotecnica e de Comp., Inst. Sistemas e Rob., Faculdade de Eng. \\ Universidade do Porto, Rua Dr. Roberto Frias 4200 465, Porto, Portugal, mrpinho@fe.up.pt \\ † Institute of Mathematics, Technical University Ilmenau, Weimarer Straße 25, 98693 Ilmenau, FRG, \\ ilchmann@mathematik.tu-ilmenau.de
}

\begin{abstract}
An optimal control problem for time-varying, nonlinear differential equations with statedependent control constraints is considered, the data may be nonsmooth. A weak maximum principle is derived in case of equality mixed constraints and pointwise set constraints imposed only on some components of the control variable. This result is then applied to derive another weak maximum principle for problems with both equality and inequality mixed constraints. The essential assumption is a full rankness condition imposed on the constraints. Additionally, a lemma shows how the results are related to previous contributions.
\end{abstract}

Key words and phrases: Optimal control, mixed constraints, maximum principle, nonsmooth analysis, weak minimizers.

\section{Introduction}

Optimality conditions for control problems with mixed state-control constraints have been the focus of attention for a long time. In particular, the subject of necessary conditions in the form of maximum principles have been addressed by a number of authors; see for example [1], [2], [3], [4], to name but a few. Weak maximum principles, which apply to weak local solutions, covering problems with possibly nonsmooth data, have been considered in [5] and, in a more general setting, in [3]. For nonsmooth problems, strong maximum principles, which in turn apply to "strong" local solutions, have also received some attention recently (see [6] and [7]).

Various recent results, including those of the present paper, can be captured as special cases of the following optimal control problem with mixed constraints, also known as state-dependent 
control constraints:

$$
\text { (P) }\left\{\begin{array}{rlrl}
\text { Minimize } l(x(a), x(b)) & & \\
\text { subject to } & & \\
\dot{x}(t) & =f(t, x(t), u(t), v(t)) & & \text { for a.a. } t \in[a, b] \\
0 & =b(t, x(t), u(t), v(t)) & & \text { for a.a. } t \in[a, b] \\
0 & \geq g(t, x(t), u(t), v(t)) & & \text { for a.a. } t \in[a, b] \\
(u(t), v(t)) & \in U(t) \times V(t) & & \text { for a.a. } t \in[a, b] \\
(x(a), x(b)) & \in C & &
\end{array}\right.
$$

where $l: \mathbb{R}^{n} \times \mathbb{R}^{n} \rightarrow \mathbb{R}, f:[a, b] \times \mathbb{R}^{n} \times \mathbb{R}^{k_{u}} \times \mathbb{R}^{k_{v}} \rightarrow \mathbb{R}^{n}, b:[a, b] \times \mathbb{R}^{n} \times \mathbb{R}^{k_{u}} \times \mathbb{R}^{k_{v}} \rightarrow \mathbb{R}^{m_{b}}$, $g:[a, b] \times \mathbb{R}^{n} \times \mathbb{R}^{k_{u}} \times \mathbb{R}^{k_{v}} \rightarrow \mathbb{R}^{m_{g}}, U:[a, b] \rightarrow \mathbb{R}^{k_{u}}$ and $V:[a, b] \rightarrow \times \mathbb{R}^{k_{v}}$ are given multifunctions and $C \subset \mathbb{R}^{n} \times \mathbb{R}^{n}$ a given set. Throughout this paper we assume that $k_{u}+k_{v} \geq m_{b}+m_{g}$.

We seek optimality necessary conditions in the form of a weak maximum principle which apply to various special cases of problem $(P)$. We are particularly interested in generalizing classical results (see [1] and [2]) to cover problems with possibly nonsmooth data.

Usually one has $m_{b} \geq 1, m_{g} \geq 1$ and, for all $t \in[a, b], U(t) \subset \mathbb{R}^{k_{u}}$ or $V(t) \subset \mathbb{R}^{k_{v}}$. However, we allow for $m_{b}, m_{g}=0, U(t)=\mathbb{R}^{k_{u}}$, or $V(t)=\mathbb{R}^{k_{v}}$ to signify the case where there are no explicit equality or inequality state-control constraints or no pointwise set constraints on some components of the control variable.

Weak local solutions are defined as follows.

Definition 1.1 A process $(\bar{x}, \bar{u}, \bar{v})$ of $(\mathrm{P})$, i.e., a triple of an absolutely continuous function $\bar{x}:[a, b] \longrightarrow \mathbb{R}^{n}$ and Lebesgue measurable functions $\bar{u}:[a, b] \longrightarrow \mathbb{R}^{k_{u}}, \bar{v}:[a, b] \longrightarrow \mathbb{R}^{k_{v}}$ satisfying the constraints of $(\mathrm{P})$, is called a weak local minimizer if, and only if, there exists some $\varepsilon>0$, such that it minimizes the cost over all processes $(x, u, v)$ of $(\mathrm{P})$ which satisfy

$$
(x(t), u(t), v(t)) \quad \in \quad T_{\varepsilon}(t), \quad \text { for a.a. } t \in[a, b]
$$

where

$$
T_{\varepsilon}(t)=(\bar{x}(t)+\varepsilon \bar{B}) \times(U(t) \cap(\bar{u}(t)+\varepsilon \bar{B})) \times(V(t) \cap(\bar{v}(t)+\varepsilon \bar{B})),
$$

and $\bar{B}$ denotes the closed unit ball.

A standard approach to obtain necessary conditions for optimal control problems involving mixed constraints in the form of inequalities is as follows. Derive conditions for problems with only equality constraints which are then applied to an "auxiliary problem" associated with (P) where inequalities are transformed into equalities by control augmentation. In fact, the inequality

$$
g(t, x(t), u(t)) \leq 0
$$


can be replaced by equality constraints and pointwise set constraints on the control variable by

$$
g(t, x(t), u(t))+v(t)=0 \quad \text { and } \quad v(t) \geq 0 .
$$

Necessary conditions have previously been derived assuming that a certain matrix $F(t)$ has full rank in the sense that $\operatorname{det} F(t) F(t)^{T} \geq L$ for a.a. $t \in[a, b]$, for some $L>0$. The full rankness condition has been imposed on the Jacobi matrix (see [1] and [2])

$$
\nabla_{u}[b, g](t, \bar{x}(t), \bar{u}(t), \bar{v}(t))
$$

or on the matrix (see [8])

$$
\Phi(t)=\left[\begin{array}{c}
b_{u}(t, \bar{x}(t), \bar{u}(t), \bar{v}(t)) \\
\mathcal{I}_{\beta}(t) \\
g_{u}(t, \bar{x}(t), \bar{u}(t), \bar{v}(t))
\end{array}\right], \mathcal{I}_{\beta}(t)=\left\{i \in\left\{1, \ldots, m_{g}\right\} \mid g_{i}(t, \bar{x}(t), \bar{u}(t), \bar{v}(t)) \geq-\beta\right\}
$$

where $\beta>0$ and $g_{u}^{\mathcal{I}_{\beta}(t)}(t, \bar{x}(t), \bar{u}(t), \bar{v}(t))$ denotes the matrix we obtain after removing from $g_{u}(t, \bar{x}(t), \bar{u}(t), \bar{v}(t))$ all the rows of index $i \notin \mathcal{I}_{\beta}(t)$.

Such rankness conditions, together with conditions enforcing continuity of the data with respect to $t$, permit the application of classical Implicit Function theorems, thereby allowing the removal of the state-dependent control constraints.

An exception is to be found in a paper by Pales and Zeidan [3]. They prove a multiplier rule for an abstract nonsmooth problem with mixed and pure constraints, and then derive necessary conditions. In the absence of pure state constraints, the weak maximum principle obtained in this way is validated with the full rankness imposed on

$$
\Upsilon(t)=\left[\begin{array}{cc}
b_{u}(t, \bar{x}(t), \bar{u}(t), \bar{v}(t)) & 0 \\
g_{u}(t, \bar{x}(t), \bar{u}(t), \bar{v}(t)) & \operatorname{diag}\left\{-g_{i}(t, \bar{x}(t), \bar{u}(t), \bar{v}(t))\right\}_{i \in\left\{1, \ldots, m_{g}\right\}}
\end{array}\right] .
$$

In the present paper we prove, first, a weak maximum principle for optimal control problems with equality mixed constraints $\left(m_{g}=0\right)$ and pointwise set constraints imposed only on some components of the control variable $\left(U(t)=\mathbb{R}^{k_{u}}, k_{v} \geq 1\right)$ following the standard approach. Since a previous weak maximum principle for nonsmooth problems involving equality constraints [5] holds in the absence of pointwise set constraints in the control variable, we extend such result to treat problems for which some pointwise set contraints on the control are also present. Different to previous work, we assume only measurability of the data with respect to $t$. Thus, a sharpened variant of the Implicit Function Theorem, a Uniform Implicit Function Theorem previously obtained in [9], must be used. Secondly, we provide a weak maximum principle for the particular case that $U(t)=\mathbb{R}^{k_{u}}$ in $(P)$ under the full rankness condition of the matrix (1.4) as in [3]. By contrast, we consider pointwise set constraints in some components of the control variable $w=(u, v)$ and relax some of the smoothness assumptions on the dynamics.

Finally, a technical lemma is given and used to compare the different full rankness conditions 
on the literature. In the presence of both inequality and equality state-control constraints, a restriction on the generality of our results is the assumption that $U(t)=\mathbb{R}^{k_{u}}$. Nonetheless , these problems are of interest when higher order conditions are considered.

A notable feature of the proofs in this paper is that they provide a simple and transparent derivation of necessary conditions, which might also be worth knowing for classical second order conditions.

\section{Preliminaries}

The notation $r \geq 0$ means that each component $r_{i}$ of $r \in \mathbb{R}^{r}$ is nonnegative. $\langle\cdot, \cdot\rangle$ denotes the Euclidean scalar product on finite dimensional vector space $\mathbb{R}^{k},|\cdot|=\sqrt{\langle\cdot, \cdot\rangle}$ the Euclidean norm, and $|\cdot|$ the induced matrix norm on $\mathbb{R}^{m \times k}$. We set

$$
\mathbb{R}_{\geq 0}^{m}=\left\{x \in \mathbb{R}^{m} \mid \quad x_{i} \geq 0 \quad \text { for } i=1, \ldots, m\right\}
$$

and $\mathbb{R}_{\leq 0}^{m}$ accordingly. The Euclidean distance function with respect to $A \subset \mathbb{R}^{k}$ is

$$
d_{A}: \mathbb{R}^{k} \rightarrow \mathbb{R}, \quad y \mapsto d_{A}(y)=\inf \{|y-x|: x \in A\} .
$$

We will often refer to the control variable as being $w$ whenever we do not want to distinguish between components. In that case, a weak local minimizer will be denote by $(\bar{x}, \bar{w})$, the control set will be $W(t) \subset \mathbb{R}^{k}, k=k_{u}+k_{v}$, and the set (1.1) will be written as

$$
T_{\varepsilon}(t)=(\bar{x}(t)+\varepsilon \bar{B}) \times(W(t) \cap(\bar{w}(t)+\varepsilon \bar{B})) .
$$

The linear space $W^{1,1}\left([a, b] ; \mathbb{R}^{p}\right)$ denotes the space of absolutely continuous functions, $L^{1}\left([a, b] ; \mathbb{R}^{p}\right)$ the space of integrable functions and $L^{\infty}\left([a, b] ; \mathbb{R}^{p}\right)$ the space of essentially bounded functions from $[a, b]$ to $\mathbb{R}^{p}$, respectively.

The following variant of a Uniform Implicit Function Theorem says that if $\phi\left(t, x_{0}(t), u_{0}(t)\right)=0$ almost everywhere, then an implicit function $\varphi(t, u)$ exists and the same neighborhood of $u_{0}$ can be chosen for all $t$. This will be essential in our setup.

Proposition 2.1 (Uniform Implicit Function Theorem) ([9])

Consider a set $T \subset \mathbb{R}^{k}$, a number $\alpha>0$, a family of functions $\left\{\psi_{a}: \mathbb{R}^{m} \times \mathbb{R}^{n} \rightarrow \mathbb{R}^{n}\right\}_{a \in T}$ and a point $\left(u_{0}, v_{0}\right) \in \mathbb{R}^{m} \times \mathbb{R}^{n}$ such that $\psi_{a}\left(u_{0}, v_{0}\right)=0$ for all $a \in T$. Assume that:

(i) $\psi_{a}$ is continuously differentiable on $\left(u_{0}, v_{0}\right)+\alpha B$ for all $a \in T$.

(ii) There exists a monotone increasing function $\theta:(0, \infty) \rightarrow(0, \infty)$ with $\theta(s) \downarrow 0$ as $s \downarrow 0$ such that, for all $a \in T,\left(u^{\prime}, v^{\prime}\right),(u, v) \in\left(u_{0}, v_{0}\right)+\alpha B$,

$$
\left|\nabla \psi_{a}\left(u^{\prime}, v^{\prime}\right)-\nabla \psi_{a}(u, v)\right| \leq \theta\left(\left|\left(u^{\prime}, v^{\prime}\right)-(u, v)\right|\right) .
$$


(iii) $\nabla_{v} \psi_{a}\left(u_{0}, v_{0}\right)$ is nonsingular for each $a \in T$ and there exists $c>0$ such that, for all $a \in T$,

$$
\left|\left[\nabla_{v} \psi_{a}\left(u_{0}, v_{0}\right)\right]^{-1}\right| \leq c
$$

Then there exist $\delta \geq 0$ and a family of continuously differentiable functions

$$
\left\{\phi_{a}: u_{0}+\delta B \rightarrow v_{0}+\alpha B\right\}_{a \in T}
$$

which are Lipschitz continuous with common Lipschitz constant $k$, such that, for all $a \in T$,

$$
\begin{aligned}
v_{0} & =\phi_{a}\left(u_{0}\right) & \\
\psi_{a}\left(u, \phi_{a}(u)\right) & =0 & \text { for all } u \in u_{0}+\delta B \\
\nabla_{u} \phi_{a}\left(u_{0}\right) & =-\left[\nabla_{v} \psi_{a}\left(u_{0}, v_{0}\right)\right]^{-1} \nabla_{u} \psi_{a}\left(u_{0}, v_{0}\right) . &
\end{aligned}
$$

The numbers $\delta$ and $k$ depend on $\theta, c$ and $\alpha$ only.

Furthermore, if $T$ is a Borel set and $a \mapsto \psi_{a}(u, v)$ is a Borel measurable function for each $(u, v) \in\left(u_{0}, v_{0}\right)+\alpha B$, then $a \mapsto \phi_{a}(u)$ is a Borel measurable function for each $u \in u_{0}+\delta B$.

We make use of the following concepts from nonsmooth analysis.

Definition 2.2 Let $A \subset \mathbb{R}^{k}$ be a closed set and $x \in A$. $p \in \mathbb{R}^{k}$ is a limiting normal to $A$ at $x$ if, and only if, there exist $p_{i} \rightarrow p$ and $x_{i} \rightarrow x$, and a sequence of positive scalars $\left\{M_{i}\right\}_{i \in \mathbb{N}}$, such that

$$
\left\langle p_{i}, x-x_{i}\right\rangle \leq M_{i}\left|x-x_{i}\right|^{2} \quad \text { for all } x \in A \text { and for each } i \in \mathbb{N}
$$

(i.e., limiting normals are limits of vectors which support $A$ at points near $x$, to second-order). The limiting normal cone to $A$ at $x$, written $N_{A}(x)$, is the set of all limiting normals to $A$ at $x$.

Given a lower semicontinuous function $f: \mathbb{R}^{k} \rightarrow \mathbb{R} \cup\{+\infty\}$ and a point $x \in \mathbb{R}^{k}$ such that $f(x)<+\infty$, the limiting subdifferential of $f$ at $x$, written $\partial f(x)$, is the set

$$
\partial f(x):=\left\{\zeta \mid(-1, \zeta) \in N_{e p i\{f\}}(f(x), x)\right\},
$$

where epi $\{f\}=\{(\eta, x) \mid \eta \geq f(x)\}$ denotes the epigragh set .

The above concepts of limiting normal cone and limiting subdifferential were first introduced in [10]. The full calculus for these constructions in finite dimensions are described in [11] and [12]. In the case that the function $f$ is Lipschitz continuous near $x$, the convex hull of the limiting subdifferential, co $\partial f(x)$, coincides with the (Clarke) generalized gradient, which may be defined directly. Properties of generalized gradients (upper semi-continuity, sum rules, etc.), are described in [13].

Throughout this paper we will refer to the following set of hypotheses which make reference to a process $(\bar{x}, \bar{w})$ of $(\mathrm{P})$ and some scalar $\varepsilon>0$ : 
(H1) $f(\cdot, x, w)$ is measurable for each $(x, w)$ and $f(t, \cdot, \cdot)$ is Lipschitz continuous with Lipschitz constant $k_{f}(t)$ on $T_{\varepsilon}(t)$ for almost all $t \in[a, b]$ and $k_{f}$ is an $L^{1}$-function.

(H2) The cost $l$ is Lipschitz continuous on a neighborhood of $(\bar{x}(a), \bar{x}(b))$ and $C$ is closed.

(H3) graph $W(\cdot)$ is Borel measurable and $W(t) \bigcap(\bar{w}(t)+\varepsilon \bar{B})$ is closed for almost all $t \in[a, b]$.

The following weak maximum principle for optimal control problems, provided in [14], will be of importance in our analysis.

Proposition 2.3 Let $\varepsilon>0$ and $(\bar{x}, \bar{w})$ denote a weak local minimizer for $(\mathrm{P})$ with restrictions $m_{b}=m_{g}=k_{v}=0$. If (H1)-(H3) are satisfied and $H(t, x, p, u)=\langle p, f(t, x, u)\rangle$ defines the Hamiltonian, then there exist $\lambda \geq 0, p \in W^{1,1}\left([a, b] ; \mathbb{R}^{n}\right)$ and $\zeta \in L^{1}\left([a, b] ; \mathbb{R}^{k_{u}}\right)$ such that, for almost all $t \in[a, b]$,

$$
\begin{aligned}
\lambda+\|p(\cdot)\|_{L^{\infty}} & \neq 0 \\
(-\dot{p}(t), \dot{\bar{x}}(t), \zeta(t)) & \in \operatorname{co} \partial H(t, \bar{x}(t), p(t), \bar{u}(t)) \\
\zeta(t) & \in \operatorname{co~} N_{U(t)}(\bar{u}(t)) \\
(p(a),-p(b)) & \in N_{C}(\bar{x}(a), \bar{x}(b))+\lambda \partial l(\bar{x}(a), \bar{x}(b)),
\end{aligned}
$$

where $\partial H$ denotes the subgradient in the $(x, p, u)$ variables.

\section{Main Results}

To simplify notation, $\bar{\phi}(t)$ will denote the evaluation of a function $\phi$ at $(t, \bar{x}(t), \bar{u}(t), \bar{v}(t))$, where $\phi$ may be $f, b, g$ or its derivatives.

Let $\mathcal{I}_{a}(t)$ be the set of indexes of the active constraints, i.e.,

$$
\mathcal{I}_{a}(t)=\left\{i \in\left\{1, \ldots, m_{g}\right\} \mid g_{i}(t, \bar{x}(t), \bar{u}(t), \bar{v}(t))=0\right\}
$$

and its complement, the set of indexes of the inactive constraints,

$$
\mathcal{I}_{c}(t)=\left\{1, \ldots, m_{g}\right\} \backslash \mathcal{I}_{a}(t)
$$

$q_{a}(t)$ denotes the cardinal of $\mathcal{I}_{a}(t)$ and $q_{c}(t)$ be the cardinal of $\mathcal{I}_{c}(t)$. Let

$$
g_{u}^{\mathcal{I}_{a}(t)}(t, \bar{x}(t), \bar{u}(t), \bar{v}(t)) \in \mathbb{R}^{q_{a}(t) \times k_{u}}
$$

(if $q_{a}(t)=0$, then the latter holds vacuously) denote the matrix we obtain after removing from $g_{u}(t, \bar{x}(t), \bar{u}(t), \bar{v}(t))$ all the rows of index $i \in \mathcal{I}_{c}(t)$.

We shall invoke the following additional hypotheses on $(P)$ : 
(H4) $b(\cdot, x, u, v)$ and $g(\cdot, x, u, v)$ are measurable for each $(x, u, v)$. For almost all $t \in[a, b]$, $b(t, \cdot, \cdot, \cdot)$ and $g(t, \cdot, \cdot, \cdot)$ are continuously differentiable functions on $(\bar{x},(t), \bar{u}(t), \bar{v}(t))+\varepsilon \bar{B}$.

There exists an increasing function $\tilde{\theta}: \mathbb{R}^{+} \rightarrow \mathbb{R}^{+}, \tilde{\theta}(s) \downarrow 0$ as $s \downarrow 0$, such that, for all $\left(x^{\prime}, u^{\prime}, v^{\prime}\right),(x, u, v) \in(\bar{x},(t), \bar{u}(t), \bar{v}(t))+\varepsilon \bar{B}$ and for almost all $t \in[a, b]$,

$$
\left|\nabla_{x, u, v}[b, g]\left(t, x^{\prime}, u^{\prime}, v^{\prime}\right)-\nabla_{x, u, v}[b, g](t, x, u, v)\right| \leq \tilde{\theta}\left(\left|\left(x^{\prime}, u^{\prime}, v^{\prime}\right)-(x, u, v)\right|\right) .
$$

There exists $K_{b, g}>0$ such that, for almost all $t \in[a, b]$,

$$
\left|\nabla_{x}[b, g](t, \bar{x}(t), \bar{u}(t), \bar{v}(t))\right|+\left|\nabla_{u}[b, g](t, \bar{x}(t), \bar{u}(t), \bar{v}(t))\right|+\left|\nabla_{v}[b, g](t, \bar{x}(t), \bar{u}(t), \bar{v}(t))\right| \leq K_{b, g} .
$$

(H5) There exists $K>0$ such that

$$
\operatorname{det}\left\{\Upsilon(t) \Upsilon^{T}(t)\right\} \geq K, \quad \text { for almost all } t \in[a, b],
$$

where $\Upsilon(t)$ is defined in (1.4).

The hypotheses (H4) and (H5) permit the application of a Uniform Implicit Function theorem as presented in Proposition 2.1. Hypothesis (H4) mainly states that the derivatives of $b$ and $g$ with respect to state and control must be uniformly continuous on a tube around the optimal solution and be bounded along the optimal solution. A full rankness condition is ensured by (H5). Further illustration of (H5) will be given in Lemma 3.3.

Theorem 3.1 (Weak Maximum Principle for $(P)$ without inequality contraints)

Let $(\bar{x}, \bar{u}, \bar{v})$ be a weak local minimizer for

$$
\left(\mathbf{P}_{=}\right)\left\{\begin{array}{rlrl}
\text { Minimize } l(x(a), x(b)) & & \\
\text { subject to } & & \\
\dot{x}(t) & =f(t, x(t), u(t), v(t)) & & \text { for a.a. } t \in[a, b] \\
0 & =b(t, x(t), u(t), v(t)) & & \text { for a.a. } t \in[a, b] \\
(u(t), v(t)) & \in \mathbb{R}^{k_{u}} \times V(t) & & \text { for a.a. } t \in[a, b] \\
(x(a), x(b)) & \in C . & &
\end{array}\right.
$$

Assume that, for some $\varepsilon>0$, hypotheses (H1)-(H5) are satisfied. Note that the matrix $\Upsilon(t)$ in (H5) simplifies to $\Upsilon(t)=b_{u}(t, \bar{x}(t), \bar{u}(t), \bar{v}(t))$ and the Hamiltonian is

$$
H(t, x, p, q, u, v)=\langle p, f(t, x, u, v)\rangle+\langle q, b(t, x, u, v)\rangle .
$$

Then there exist $p \in W^{1,1}\left([a, b] ; \mathbb{R}^{n}\right), \zeta \in L^{1}\left([a, b] ; \mathbb{R}^{k_{v}}\right)$ and $\lambda \geq 0$ such that, for almost all $t \in[a, b]$,

$$
\begin{aligned}
\|p\|_{L_{\infty}}+\lambda & \neq 0 \\
(-\dot{p}(t), 0, \zeta(t)) & \in \operatorname{co} \partial_{x, u, v} H(t, \bar{x}(t), p(t), q(t), \bar{u}(t), \bar{v}(t)) \\
\zeta(t) & \in \operatorname{co} N_{V(t)}(\bar{v}(t)) \\
(p(a),-p(b)) & \in N_{C}(\bar{x}(a), \bar{x}(b))+\lambda \partial l(\bar{x}(a), \bar{x}(b))
\end{aligned}
$$


Furthermore, there exists an $M>0$ such that

$$
\left|\left(\Upsilon(t) \Upsilon(t)^{T}\right)^{-1}\right| \leq M \quad \text { for a.a. } t \in[a, b]
$$

and

$$
|q(t)| \leq k_{f}(t) M K_{b, g}|p(t)| \quad \text { for a.a. } t \in[a, b]
$$

The proof of Theorem 3.1 is given in Section 4 .

We now turn to optimal control problems with mixed state-control constraints in the form of equalities and inequalities and we assume that $U(t)=\mathbb{R}^{k_{u}}$.

Theorem 3.2 (Weak Maximum Principle for $(P)$ with $U(t)=\mathbb{R}^{k_{u}}$ )

Let $(\bar{x}, \bar{u}, \bar{v})$ be a weak local minimizer for $(\mathrm{P})$ with $U(t)=\mathbb{R}^{k_{u}}$. Assume that, for some $\varepsilon>0$, hypotheses (H1)-(H5) are satisfied and that $g(\cdot, \bar{x}(\cdot), \bar{u}(\cdot), \bar{v}(\cdot))$ is bounded on $[a, b]$. Then there exist $p \in W^{1,1}\left([a, b] ; \mathbb{R}^{n}\right), \eta(t) \in L^{1}\left([a, b], \mathbb{R}^{k_{v}}\right)$ and $\lambda \geq 0$ such that, for almost all $t \in[a, b]$,

$$
\begin{aligned}
\|p\|_{L_{\infty}}+\lambda & \neq 0, \\
(-\dot{p}(t), 0, \eta(t)) & \in \operatorname{co} \partial_{x, u, v} H(t, \bar{x}(t), p(t), q(t), r(t), \bar{u}(t), \bar{v}(t)), \\
\eta(t) & \in \operatorname{co} N_{V(t)}(\bar{v}(t)), \\
\langle r(t), g(t, \bar{x}(t), \bar{u}(t), \bar{v}(t))\rangle & =0 \\
(p(a),-p(b)) & \in N_{C}(\bar{x}(a), \bar{x}(b))+\lambda \partial l(\bar{x}(a), \bar{x}(b)) .
\end{aligned}
$$

Furthermore, there exists an $M>0$ such that

$$
|(q(t), r(t))| \leq k_{f}(t) M K_{b, g}|p(t)| \quad \text { for a.a. } t \in[a, b] .
$$

The proof of Theorem 3.2 is given in Section 4 .

If $k_{v}=0$ and $g$ and $b$ do not depend on $x$, we recover the weak Maximum Principle for standard Optimal Control with pointwise set constraints in the control variable

$$
u(t) \in U(t)=\left\{u \in \mathbb{R}^{k} \mid b_{i}(t, u)=0 \text { and } g_{j}(t, u) \leq 0, i, j=1, \ldots, m_{g}\right\} \quad \text { for a.a. } t \in[a, b] .
$$

The following lemma and remark will be used in clarifying the meaning of (H5) and other assumptions made in the literature.

Lemma 3.3 Let $k, n \in \mathbb{N}$ such that, for all $t \in[a, b], m(t), q(t), l(t) \in \mathbb{N}_{0}, m(t)+q(t)=n \leq k$, and consider

$$
J(t):=\left[\begin{array}{ccc}
A(t) & 0 & 0 \\
N(t) & 0 & B(t)
\end{array}\right] \in \mathbb{R}^{n \times(k+l(t)+q(t))},
$$


with

$$
A(t) \in \mathbb{R}^{m(t) \times k}, N(t) \in \mathbb{R}^{q(t) \times k}, B(t) \in \mathbb{R}^{q(t) \times q(t)} .
$$

Then the following conditions

(i) $\exists c_{A}>0: \operatorname{det} A(t) A(t)^{T} \geq c_{A}$ for a.a. $t \in[a, b]$

(ii) $\exists c_{B}>0: \operatorname{det} B(t) B(t)^{T} \geq c_{B}$ for a.a. $t \in[a, b]$

(iii) $\exists c>0: \operatorname{det} J(t) J(t)^{T} \geq c \quad$ for a.a. $t \in[a, b]$

are related as follows

- (i), (ii) $\wedge N \in L^{\infty} \quad \Longrightarrow \quad$ (iii),

- (iii) $\Longrightarrow$ (i),

- (iii) $\stackrel{\text { i.g. }}{\rightleftharpoons}$ (ii).

Remark 3.4 In what follows, we assume that the data satisfies (H4).

(a) Using the abbreviations introduced at the beginning of Section 3, we set

$$
\begin{aligned}
A(t) & =\left[\begin{array}{c}
b_{u}(t, \bar{x}(t), \bar{u}(t), \bar{v}(t)) \\
g_{u}^{\mathcal{I}_{a}(t)}(t, \bar{x}(t), \bar{u}(t), \bar{v}(t))
\end{array}\right] \in \mathbb{R}^{\left(m_{g}+q_{a}(t)\right) \times k_{u}}, \\
N(t) & =g_{u}^{\mathcal{I}_{c}(t)}(t, \bar{x}(t), \bar{u}(t), \bar{v}(t)) \in \mathbb{R}^{q_{c}(t) \times k_{u}}, \quad B(t)=\operatorname{diag}\left\{-\bar{g}_{i}(t)\right\}_{i \in \mathcal{I}_{c}(t)} \in \mathbb{R}^{q_{c}(t) \times q_{c}(t),}, \\
\text { and } m_{g} & =q_{a}(t)+q_{c}(t) .
\end{aligned}
$$

If the components of $g$ are permuted in such a way that the active contraints come first, then $\Upsilon(t)$ as defined in (1.4) and $J(t)$ as in Lemma 3.3 coincide and we have

$$
\Upsilon(t)=J(t)=\left[\begin{array}{lll}
b_{u}(t, \bar{x}(t), \bar{u}(t), \bar{v}(t)) & 0 & 0 \\
\mathcal{I}_{u}^{\mathcal{I}_{u}(t)}(t, \bar{x}(t), \bar{u}(t), \bar{v}(t)) & & \\
\mathcal{I}_{u}^{\mathcal{I}_{c}(t)}(t, \bar{x}(t), \bar{u}(t), \bar{v}(t)) & 0 & \operatorname{diag}\left\{-\bar{g}_{i}(t)\right\}_{i \in \mathcal{I}_{c}(t)}
\end{array}\right] \in \mathbb{R}^{\left(m_{b}+m_{g}\right) \times\left(k_{u}+m_{g}\right)}
$$

Note that the permutaion of the components of $g$ depends on $t$ but does not change a full rankness condition. Suppose there exists some $L>0$ such that

$$
\operatorname{det}\left\{\nabla_{u}[b, g](t, \bar{x}(t), \bar{u}(t), \bar{v}(t)) \nabla_{u}[b, g](t, \bar{x}(t), \bar{u}(t), \bar{v}(t))^{T}\right\} \geq L \quad \text { for a.a. } \quad t \in[a, b] .
$$

Then

$$
\nabla_{u}[b, g](t, \bar{x}(t), \bar{u}(t), \bar{v}(t))=\left[\begin{array}{c}
A(t) \\
N(t)
\end{array}\right]
$$

and an application of Weyl's Theorem (see e.g. [15]) yields condition (iii) of Lemma 3.3, or equivalently (H5). 
(b) If there exists some $L>0$ such that

$$
\operatorname{det}\left\{\Phi(t) \Phi(t)^{T}\right\} \geq L \quad \text { for a.a. } t \in[a, b]
$$

where $\Phi(t)$ is defined in (1.3), then we show that condition (iii) of Lemma 3.3 also follows: Set $\tilde{\mathcal{I}}_{\beta}(t)=\left\{1, \ldots, m_{g}\right\} \backslash \mathcal{I}_{\beta}(t)$ and, using the notation of Lemma 3.3,

$$
\begin{aligned}
& A(t)=\left[\begin{array}{cc}
\Phi(t) & 0 \\
N(t) & =\left[g_{u}^{\tilde{\mathcal{I}}_{\beta}(t)}(t, \bar{x}(t), \bar{u}(t), \bar{v}(t)), 0\right],
\end{array}\right], \\
&
\end{aligned}
$$

Applying again Weyl's Theorem to $A(t) A(t)^{T}$ and invoking the full rankness condition of $\Phi(t)$ shows that $A(t)$ satisfies (i) of Lemma 3.3. By definition of $\tilde{\mathcal{I}}_{\beta}(t), B(t)$ satisfies (ii) of Lemma 3.3. Since $N \in L^{\infty}$ we conclude that (iii) of Lemma 3.3.

(c) Sufficient conditions for (H5) to hold are given in (a) and (b) above. Conversely, if (H5), or equivalently condition (iii) of Lemma 3.3, is satisfied, then condition (i) of Lemma 3.3 holds true. However, there are systems for which (i) of Lemma 3.3 holds but (iii) does not. Since, by (H4), $N \in L^{\infty}$, candidates are systems, for which (iii) is not valid. In Example 3.5 we provide such an optimal control problem for which condition (i) of Lemma 3.3 holds but not (iii). The interest of the example resides on the simple observation that the data of the problem does not satisfy (H5) but, nevertheless, Theorem 3.2 provides a nontrivial set of multipliers associated with the optimal solution of it. This does not come as a surprise. In fact, since the components of the multiplier $r$ are zero when $i \in \mathcal{I}_{c}(t)$, the derivative with respect to $u$ of the corresponding components of $g$ do not take any part in the determination of the multipliers. One could then conjecture that Theorem 3.2 would hold when (H5) is replaced by merely the full rankness of $A(t)$ as defined above in (a). To our knowledge, derivation of optimality conditions for $(\mathrm{P})$ under merely such assumption (the full rankness of the derivative with respect to $u$ of the active constraints only) is an open problem.

Example 3.5 Consider the problem of minimizing $x(1)$ subject to

$$
\begin{aligned}
& \dot{x}(t)=u_{1}^{2}+u_{2}^{2}+u_{3}^{2} \quad \text { for a.a. } t \in[0,1] \\
& 0 \geq u_{1}+u_{2} u_{3} \quad \text { for a.a. } t \in[0,1] \\
& 0 \geq t u_{1}+u_{2} u_{3}-t \quad \text { for a.a. } t \in[0,1] \\
& x(0)=0 .
\end{aligned}
$$

It is easy to check that $\left(\bar{x}=0,\left(\bar{u}_{1}, \bar{u}_{2}, \bar{u}_{3}\right)=0\right)$ is a minimizer for such problem and

$$
\mathcal{I}_{a}(t)=\left\{\begin{array}{lll}
\{1\} & \text { if } & t \neq 0 \\
\{1,2\} & \text { if } & t=0
\end{array}\right.
$$


Using the notation of Lemma 3.3 we have,

$$
\begin{aligned}
& A(t)= \begin{cases}{[1,0,0],} & t \in(0,1] \\
{\left[\begin{array}{lll}
1 & 0 & 0 \\
0 & 0 & 0
\end{array}\right],} & t=0\end{cases} \\
& N(t)=\left\{\begin{array}{ll}
{[t, 0,0],} & t \in(0,1] \\
\text { does not exist, } & t=0
\end{array}, B(t)= \begin{cases}-t, & t \in(0,1] \\
\text { does not exist, } & t=0 .\end{cases} \right.
\end{aligned}
$$

Note that $A(t), N(t)$, and $B(t)$ may change formats, but

$$
J(t)=\left\{\begin{array}{l}
{\left[\begin{array}{cccccc}
1 & 0 & 0 & 0 & 0 & 0 \\
t & 0 & 0 & 0 & 0 & -t
\end{array}\right], \quad t \in(0,1]} \\
{\left[\begin{array}{lllllc}
1 & 0 & 0 & 0 & 0 & 0 \\
0 & 0 & 0 & 0 & 0 & 0
\end{array}\right], \quad t=0}
\end{array}\right.
$$

does not. (i) of Lemma 3.3 is satisfied since

$$
\operatorname{det} A(t) A(t)^{T}= \begin{cases}1, & t \in(0,1] \\ 0, & t=0 .\end{cases}
$$

(ii) and (iii) do not hold since $t \mapsto J(t)$ is continuous and

$$
\operatorname{det} B(t) B(t)^{T}=\operatorname{det} J(t) J(t)^{T}=t^{2} \quad \text { for all } t \in(0,1] .
$$

Application of Theorem 3.2 to this problem provides a set of multipliers associated with it.

\section{Proofs of main results}

Theorem 3.1 is proved first. There is a parallel here with the weak maximum principle for optimal control problems involving differential algebraic equations of index 1 proved in [9] in that the proof of Theorem 3.1 is similar to Theorem 3.2 in [9]. The main step consists in rewriting the constraints so that a Uniform Implicit Function theorem, Proposition 2.1, applies. Observe that a sharpened version of an Implicit Function theorem is needed, since we work under the assumption that the data is merely measurable with respect to $t$. Then we associate with $\left(\mathrm{P}_{=}\right)$ an "auxiliary problem". This problem is a standard optimal control problem, but we must apply the nonsmooth Maximum Principle Proposition of 2.3 to yield the required conditions for (P).

Proof of Theorem 3.1: We proceed in several steps. Let $\varepsilon>0$ be as in Theorem 3.1.

Step 1: We show that the choice of $\mu:[a, b] \times\left(\mathbb{R}^{n} \times \mathbb{R}^{k_{u}} \times \mathbb{R}^{k_{v}}\right) \times \mathbb{R}^{m_{b}} \rightarrow \mathbb{R}^{m_{b}}$ given by

$$
\mu(t,(\xi, u, v), \eta):=b\left(t, \bar{x}(t)+\xi, \bar{u}(t)+u+\Upsilon(t)^{T} \eta, \bar{v}(t)+v\right)
$$


asserts, for some $\sigma \in(0, \varepsilon), \delta \in(0, \varepsilon)$, the existence of a Borel set $T=[a, b] \backslash S$, where $S$ is of measure zero, and an implicit map

$$
d: T \times \sigma B \times \sigma B \times \sigma B \longrightarrow \delta B
$$

such that $d(\cdot, \xi, u, v)$ is a measurable function for fixed $(\xi, u, v)$, the functions $\{d(t, \cdot, \cdot, \cdot) \mid t \in T\}$ are Lipschitz continuous with common Lipschitz constant, $d(t, \cdot, \cdot, \cdot)$ is continuously differentiable for fixed $t \in T$,

$$
\begin{array}{rll}
d(t, 0,0,0) & =0 & \text { for a.a. } t \in[a, b] \\
\mu(t,(\xi, u, v), d(t, \xi, u, v)) & =0 & \text { for a.a. } t \in[a, b] \text { and all }(\xi, u, v) \in \sigma B \times \sigma B \times \sigma B
\end{array}
$$

such that

$$
\left(d_{\xi}, d_{u}, d_{v}\right)(t, 0,0,0)=-\left[\Upsilon(t) \Upsilon(t)^{T}\right]^{-1}\left(\bar{b}_{x}(t), \bar{b}_{u}(t), \bar{b}_{v}(t)\right) \quad \text { for a.a. } t \in[a, b]
$$

Recall that the abbreviation of $\bar{b}_{x}(t)$ and similar was introduced at the beginning of Section 3 . Choose $S_{0} \subset[a, b]$ to be the largest subset such that each of the conditions in (H1), (H4) and (H5) do not hold for every $t \in S_{0}$. By assumption, $S_{0}$ is of Lebesgue measure zero. It follows from page 309 in [16] that there exists a Borel set $S$, which is the intersection of a countable collection of open sets, such that $S_{0} \subset S$ and $S \backslash S_{0}$ has measure zero. Thus $S$ is a Borel set of measure zero. We define $T=[a, b] \backslash S$, a Borel set of full measure, and identify $((\xi, u, v), \eta)$ with $(u, v)$ in Proposition 2.1.

Observe that

$$
\frac{\partial \mu}{\partial \eta}(t, 0,0,0,0)=\Upsilon(t) \Upsilon(t)^{T}
$$

By (H5), $\Upsilon(t) \Upsilon(t)^{T}$ is a real symmetric positive definite matrix with determinant uniformly bounded away from the origin for all $t \in T$ and thus, together with (H4), there exists $M>0$ such that (3.1) holds.

In order to apply Proposition 2.1, we identify $t$ with $a$, $\mu$ with $\psi,(0,0,0)$ with $u_{0},(\xi, u, v)$ with $u$, and $\eta$ with $v$, and so there exists an implicit map $\phi$ which here we denote by $d$.

Choose $\sigma_{1}, \delta_{1}>0$ such that

$$
\sigma_{1} \in(0, \min \{\sigma, \varepsilon / 2\}), \quad \delta_{1} \in(0, \min \{\delta, \varepsilon / 2\}), \quad \sigma_{1}+K_{b, g} \delta_{1} \in(0, \varepsilon / 2),
$$

where $\sigma$ and $\delta$ are as above and $K_{b, g}$ is given by (H5).

In what follows and without loss of generality, we consider the implicit function $d$ defined on $[a, b] \times \sigma_{1} B \times \sigma_{1} B \times \sigma_{1} B$ and taking values in $\delta_{1} B$.

Step 2: We show that if $(\bar{x}, \bar{u}, \bar{v})$ is a weak local minimizer for $\left(\mathrm{P}_{=}\right)$, then $(\bar{x}, \bar{u}, \bar{v})$ is a weak 
local minimizer for the auxiliary optimal control problem

$$
\left(\mathbf{P}_{=}^{\text {aux }}\right)\left\{\begin{array}{rlrl}
\text { Minimize } l(x(a), x(b)) & \\
\text { subject to } & & \\
\dot{x}(t) & =\phi(t, x(t), u(t), v(t)) & & \text { for a.a. } t \in[a, b] \\
(u(t), v(t)) & \in U_{\sigma_{1}}(t) \times V_{\sigma_{1}}(t) & \text { for a.a. } t \in[a, b] \\
(x(a), x(b)) & \in C, &
\end{array}\right.
$$

in which

$$
\begin{aligned}
\phi(t, x, u, v) & =f\left(t, x, u+\Upsilon(t)^{T} d(t, x-\bar{x}(t), u-\bar{u}(t), v-\bar{v}(t)), v\right), \\
U_{\sigma_{1}}(t) & =\bar{u}(t)+\sigma_{1} B \\
V_{\sigma_{1}}(t) & =V(t) \cap\left(\bar{v}(t)+\sigma_{1} B\right) .
\end{aligned}
$$

Suppose that $(\tilde{x}, \tilde{u}, \tilde{v})$ is a solution of $\left(\mathrm{P}_{=}^{\text {aux }}\right)$ with lesser cost, i.e., $l(\tilde{x}(a), \tilde{x}(b))<l(\bar{x}(t), \bar{x}(b))$. Set

$$
\begin{aligned}
\hat{u}(t) & =\tilde{u}(t)+\Upsilon(t)^{T} d(t, \tilde{x}(t)-\bar{x}(t), \tilde{u}(t)-\bar{u}(t), \tilde{v}(t)-\bar{v}(t)) \\
\xi(t) & =\tilde{x}(t)-\bar{x}(t) \\
u_{1}(t) & =\tilde{u}(t)-\bar{u}(t) \\
v_{1}(t) & =\tilde{v}(t)-\bar{v}(t) .
\end{aligned}
$$

¿From (4.2) and the definition of $d$ it follows that

$$
\begin{aligned}
|\hat{u}(t)-\bar{u}(t)| & \leq|\tilde{u}(t)-\bar{u}(t)|+K_{b, g} \delta_{1} \leq \sigma_{1}+K_{b, g} \delta_{1}<\varepsilon \\
|\tilde{v}(t)-\bar{v}(t)| & \leq \sigma_{1}<\varepsilon
\end{aligned}
$$

By the definition of $d$, for almost all $t \in[a, b]$,

$$
\mu\left(t,\left(\xi(t), u_{1}(t), v_{1}(t)\right), d\left(t, \xi(t), u_{1}(t), v_{1}(t)\right)=b(t, \tilde{x}(t), \hat{u}(t), \tilde{v}(t))=0 .\right.
$$

We conclude that $(\tilde{x}, \hat{u}, \tilde{v})$ is a solution of $\left(P_{=}\right)$with lesser cost, contradicting the optimality of $(\bar{x}, \bar{u}, \bar{v})$.

Step 3: We apply Proposition 2.3 to $\left(\mathrm{P}_{=}^{\mathrm{aux}}\right)$.

It is easy to see that the suppositions of Proposition 2.3 are satisfied. Thus, there exist $p \in$ $W^{1,1}\left([a, b] ; \mathbb{R}^{n}\right)$ and $\lambda \geq 0$ such that, for almost all $t \in[a, b]$,

$$
\begin{aligned}
& (-\dot{p}(t), \dot{\bar{x}}(t), 0,0) \\
& \quad \in \operatorname{co} \partial_{x, p, u, v}\langle p(t), \phi(t, \bar{x}(t), \bar{u}(t), \bar{v}(t))\rangle-\left[\{0\} \times\{0\} \times \operatorname{co} N_{U_{\sigma}(t)}(\bar{u}(t)) \times \operatorname{co} N_{V_{\sigma}(t)}(\bar{v}(t))\right]
\end{aligned}
$$

and

$$
(p(a),-p(b)) \in N_{C}(\bar{x}(a), \bar{x}(b))+\lambda \partial l(\bar{x}(a), \bar{x}(b))
$$


We deduce from the nonsmooth chain rule (see Theorem 2.3.9 in [13]) and the differentiability properties of $d$ the following estimate

$$
\begin{gathered}
\operatorname{co} \partial_{x, p, u, v}\langle p, \phi(t, x, u, v)\rangle=\operatorname{co} \partial_{x, p, u, v}\left\langle p(t), f\left(t, x, u+\Upsilon(t)^{T} d(t, x-\bar{x}(t), u-\bar{u}(t), v-\bar{v}(t)), v\right)\right\rangle \\
\subset\left\{\left(\nu-\rho \Gamma(t) \bar{b}_{x}(t), \gamma, \rho-\rho \Gamma(t) \bar{b}_{u}(t), \pi-\rho \Gamma(t) \bar{b}_{v}(t)\right) \mid(\nu, \gamma, \rho, \pi) \in \operatorname{co} \partial_{x, p, u, v}\langle p, f\rangle\right\}
\end{gathered}
$$

where

$$
\Gamma(t):=\Upsilon^{T}(t)\left[\Upsilon(t) \Upsilon(t)^{T}\right]^{-1}
$$

Appealing to an appropriate selection theorem, we deduce existence of measurable functions $\nu$, $\gamma, \rho, \pi$ and $\left(\zeta_{1}, \zeta_{2}\right)$ satisfying, for almost all $t \in[a, b]$,

$$
\begin{aligned}
(\nu(t), \gamma(t), \rho(t), \pi(t)) & \in \operatorname{co~} \partial_{x, p, u, v}\langle p(t), f(t, \bar{x}(t), \bar{u}(t), \bar{v}(t)\rangle \\
\left(\zeta_{1}(t), \zeta_{2}(t)\right) & \in \operatorname{co~} N_{U_{\sigma}(t)}(\bar{u}(t)) \times \operatorname{co~} N_{V_{\sigma}(t)}(\bar{v}(t))
\end{aligned}
$$

and

$$
\left(-\dot{p}(t), \dot{\bar{x}}(t),\left(\zeta_{1}(t), \zeta_{2}(t)\right)\right)=\left(\nu(t)+q(t) \bar{b}_{x}(t), \gamma(t), \rho(t)+q(t) \bar{b}_{u}(t), \pi(t)+q(t) \bar{b}_{v}(t)\right)
$$

where

$$
q(t)=-\rho(t) \Upsilon(t)^{T}\left[\Upsilon(t) \Upsilon(t)^{T}\right]^{-1}
$$

Under the hypotheses $\nu, \gamma, \rho, \pi$ and $\left(\zeta_{1}, \zeta_{2}\right)$ are all integrable functions, and so is $q$.

Step 4: We show (i)-(iv).

Since $N_{U_{\sigma}(t)}(\bar{u}(t))=\{0\}$, we see that, for $\zeta(t):=\zeta_{2}(t)$ and for almost all $t \in[a, b]$,

$$
\begin{aligned}
(-\dot{p}(t), 0, \zeta(t)) & \in \operatorname{co~} \partial_{x, u, v} H(t, \bar{x}(t), p(t), q(t), \bar{u}(t), \bar{v}(t)) \\
\zeta(t) & \in \operatorname{co~} N_{V_{\sigma}(t)}(\bar{v}(t)) .
\end{aligned}
$$

This proves that $\lambda, p, q$ and $\zeta$ satisfy (i)-(iv).

(H1) and (3.1) yield that, for all $t \in T$,

$$
|q(t)| \leq|p(t)| k_{f}(t)\left|[b, g]_{u}(t)\right| M
$$

and hence (3.2) follows from (H4). This completes the proof.

Proof of Lemma 3.3: $\quad$ Let $\mathcal{M} \subset[a, b]$ to be the largest set such that (i)-(iii) hold for all $t \in \mathcal{M}$. To simplify the notation, define for sequences $\left\{t_{i}\right\}_{i \in \mathbb{N}} \subset \mathcal{M}$ and $\left\{\left(x_{i}, y_{i}\right)\right\}_{i \in \mathbb{N}} \subset \mathbb{R}^{m\left(t_{i}\right) \times q\left(t_{i}\right)}$,

$$
\begin{aligned}
\varphi_{i}: & =\left[x_{i}^{T}, y_{i}^{T}\right]\left[\begin{array}{ccc}
A\left(t_{i}\right) & 0 & 0 \\
N\left(t_{i}\right) & 0 & B\left(t_{i}\right)
\end{array}\right]\left[\begin{array}{ccc}
A\left(t_{i}\right) & 0 & 0 \\
N\left(t_{i}\right) & 0 & B\left(t_{i}\right)
\end{array}\right]^{T}\left[\begin{array}{c}
x_{i} \\
y_{i}
\end{array}\right] \\
& =\left|A\left(t_{i}\right)^{T} x_{i}+N\left(t_{i}\right) y_{i}\right|^{2}+\left|B\left(t_{i}\right)^{T} y_{i}\right|^{2} .
\end{aligned}
$$


"(i), (ii) \& $\mathbf{N} \in \mathbf{L}^{\infty} \Longrightarrow$ (iii)": Seeking a contradiction, suppose there exists a sequence $\left\{t_{i}\right\}_{i \in \mathbb{N}} \subset \mathcal{M}$ and

$$
\left\{\left(x_{i}, y_{i}\right)\right\}_{i \in \mathbb{N}} \subset \mathbb{R}^{m\left(t_{i}\right) \times q\left(t_{i}\right)} \quad \text { with } \quad\left|\left(x_{i}^{T}, y_{i}^{T}\right)\right|=1 \quad \text { for all } i \in \mathbb{N},
$$

such that

$$
\lim _{i \rightarrow \infty} \varphi_{i}=0
$$

Suppose there are only finitely many $q\left(t_{i}\right) \geq 0$. If necessary, extract a subsequence (we do not relabel) $\left\{t_{i}\right\}_{i \in \mathbb{N}}$ so that (4.3) and (4.4) hold and $q\left(t_{i}\right)=0$ for all $i \in \mathbb{N}$. It follows that

$$
\lim _{i \rightarrow \infty} \varphi_{i}=\lim _{i \rightarrow \infty}\left|A\left(t_{i}\right)^{T} x_{i}\right|=0 \quad \text { and }\left|x_{i}\right|=1 \quad \text { for all } i \in \mathbb{N} .
$$

This violates (i) and yields a contradiction.

If there exist infinitely many $i \in \mathbb{N}$ with $q\left(t_{i}\right) \geq 1$, then extract a subsequence (again we do not relabel) $\left\{t_{i}\right\}_{i \in \mathbb{N}}$ such that $q\left(t_{i}\right) \geq 1$ for all $i \in \mathbb{N}$. If $\left\{\left|y_{i}\right|\right\}_{i \in \mathbb{N}}$ is bounded away from 0 , then, by (ii) we have $\lim _{i \rightarrow \infty} y_{i}=0$, contradicting the boundedness of $\left\{\left|y_{i}\right|\right\}_{i \in \mathbb{N}}$ away from zero. If, however, $\left\{\left|y_{i}\right|\right\}_{i \in \mathbb{N}}$ is not bounded away from 0 , then we may extract a further subsequence $\left\{t_{i}\right\}_{i \in \mathbb{N}}$ such that $\lim _{i \rightarrow \infty} y_{i}=0$. In this case (4.4) gives $\lim _{i \rightarrow \infty} A\left(t_{i}\right)^{T} x_{i}+N\left(t_{i}\right) y_{i}=0$, and, since $N$ is bounded along the subsequence, we deduce that $\lim _{i \rightarrow \infty} A\left(t_{i}\right)^{T} x_{i}=0$, whence, by (i), $\lim _{i \rightarrow \infty} x_{i}=0$. This contradicts $\left|\left(x_{i}^{T}, y_{i}^{T}\right)\right|=1$ for all $i \in \mathbb{N}$, and thus the first part of the proof is complete.

"(iii) $\Longrightarrow$ (i)": Once again, seeking a contradiction, suppose that there exists a sequence $\left\{t_{i}\right\}_{i \in \mathbb{N}} \subset \mathcal{M}$ such that

$$
\lim _{i \rightarrow \infty} A\left(t_{i}\right)^{T} x_{i}=0 \quad \text { and } \quad\left|x_{i}\right|=1 \quad \text { for all } i \in \mathbb{N} \text {. }
$$

Then, for

$$
\left(x_{i}^{T}, y_{i}^{T}\right)=\left\{\begin{array}{lll}
\left(x_{i}^{T}, 0\right), & \text { if } & q\left(t_{i}\right) \geq 1 \\
x_{i}^{T}, & \text { if } & q\left(t_{i}\right)=0
\end{array}\right.
$$

it follows that

$$
\lim _{i \rightarrow \infty} \varphi_{i}=0 \quad \text { and } \quad\left|\left(x_{i}^{T}, y_{i}^{T}\right)\right|=1 .
$$

This contradicts (iii) and completes the second part of the proof.

"(iii) $\stackrel{\text { i.g. }}{\Longrightarrow}$ (ii)": The constant matrices $A=[0,1], N=[1,0]$ and $B=0$ satisfy

$$
\left[\begin{array}{lll}
A & 0 & 0 \\
N & 0 & B
\end{array}\right]\left[\begin{array}{lll}
A & 0 & 0 \\
N & 0 & B
\end{array}\right]^{T}=\left[\begin{array}{ll}
1 & 0 \\
0 & 1
\end{array}\right]
$$

and hence (iii) is certainly satisfied but not (ii). 
Now we are in a position to prove Theorem 3.2 by applying Theorem 3.1 to a suitable auxiliary problem.

Proof of Theorem 3.2: $\quad$ In order to apply Theorem 3.1, we define an auxiliary optimal control problem.

Let $\alpha, \zeta:[a, b] \rightarrow \mathbb{R}^{m_{g}}$ be measurable functions. Define two matrices

$$
\begin{array}{ll}
E(t)=\operatorname{diag}\left\{-g_{i}(t, \bar{x}(t), \bar{u}(t), \bar{v}(t))\right\}_{i \in\left\{1, \ldots, m_{g}\right\}}, & z_{i}(t)= \begin{cases}1 & \text { if } \quad i \in \mathcal{I}_{a}(t) \\
0 & \text { if } \quad i \in \mathcal{I}_{c}(t) .\end{cases} \\
Z(t)=\operatorname{diag}\left\{z_{1}(t), \ldots, z_{m_{g}}(t)\right\},
\end{array}
$$

For $\varepsilon \in(0,1)$ and a weak local minimizer $(\bar{x}, \bar{u}, \bar{v})$ for $(\mathrm{P})$ with $U(t)=\mathbb{R}^{k_{u}}$, we consider the optimal control problem

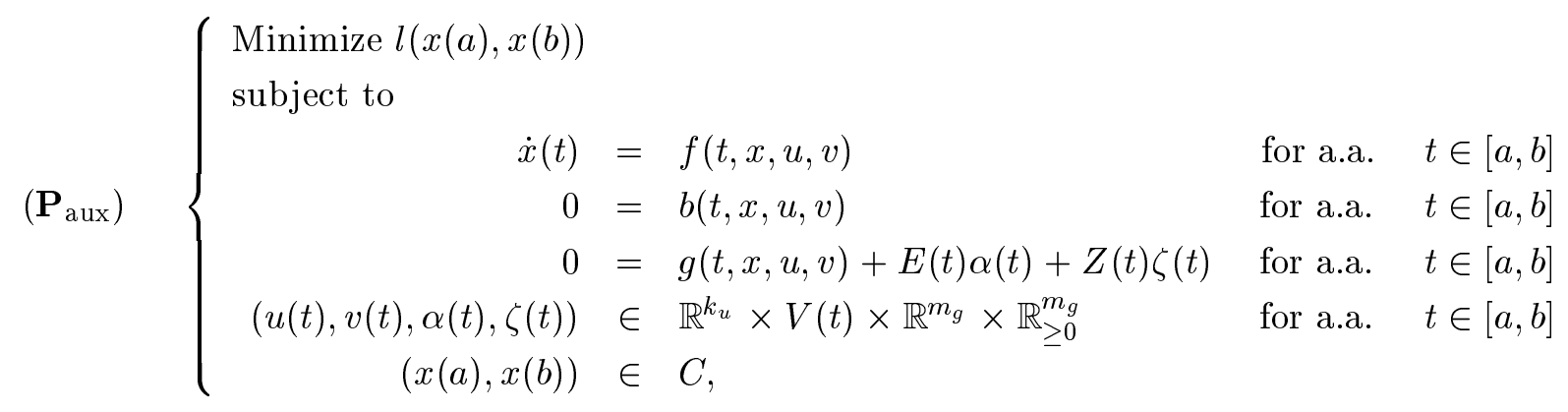

and proceed in several steps.

Step 1: We show that $(\bar{x}, \bar{u}, \bar{v}, \bar{\alpha}, \bar{\zeta})$ is a weak local minimizer for $\left(P_{\text {aux }}\right)$ where

$$
\bar{\zeta}_{i}(t)=-g_{i}(t, \bar{x}(t), \bar{u}(t), \bar{v}(t)) \quad \text { and } \quad \bar{\alpha}_{i}(t)=1 \quad \text { for } \quad i=1, \ldots, m_{g} .
$$

Suppose that there exists an admissible solution $(\tilde{x}, \tilde{u}, \tilde{v}, \tilde{\alpha}, \tilde{\zeta})$ for $\left(P_{\text {aux }}\right)$ with lesser cost. For any $i \in \mathcal{I}_{a}(t)$, we have $g_{i}(t, \tilde{x}(t), \tilde{u}(t), \tilde{v}(t))+\tilde{\zeta}_{i}(t)=0$, and since $\tilde{\zeta}_{i}(t) \geq 0$, it follows that $g_{i}(t, \tilde{x}(t), \tilde{u}(t), \tilde{v}(t)) \leq 0$. Taking into account that any $\tilde{\alpha}$ is such that $\tilde{\alpha}(t) \in \bar{\alpha}(t)+\varepsilon \bar{B}$ and that $\varepsilon<1$, we also deduce that $g_{i}(t, \tilde{x}(t), \tilde{u}(t), \tilde{v}(t)) \leq 0$, for all $i \in \mathcal{I}_{c}(t)$. This means that $(\tilde{x}, \tilde{u}, \tilde{v})$ is a solution for $(P)$ with lesser cost, contradicting the optimality of $(\bar{x}, \bar{u}, \bar{v})$.

Step 2: We prove that the data of $\left(P_{\text {aux }}\right)$ satisfies the hypotheses under which Theorem 3.1 is applicable, i.e., (H1)-(H5) for

$$
\psi(t, x(t), u(t), v(t), \alpha(t), \zeta(t))=\left(\begin{array}{c}
b(t, x, u, v) \\
g(t, x, u, v)+E(t) \alpha(t)+Z(t) \zeta(t)
\end{array}\right) \in \mathbb{R}^{m_{b}+m_{g}},
$$

$(u, \alpha)$ and $(v, \zeta)$ taking the role of $b, u$ and $v$, respectively, are satisfied.

(H1)-(H3) are immediate. To see (H5) note that the derivative of $\bar{\psi}$ with respect to $u$ and $\alpha$ is, in terms of (1.4),

$$
\bar{\psi}_{u, \alpha}(t)=\psi_{u, \alpha}(t, \bar{x}(t), \bar{u}(t), \bar{v}(t), \bar{\alpha}(t), \bar{\zeta}(t))=\Upsilon(t)
$$


It remains to show (H4). By presupposition there exists an increasing function $\tilde{\theta}: \mathbb{R}^{+} \rightarrow \mathbb{R}^{+}$, $\tilde{\theta}(s) \downarrow 0$ as $s \downarrow 0$, such that, for all $\left(x^{\prime},\left(u^{\prime}, \alpha^{\prime}\right),\left(v^{\prime}, \zeta^{\prime}\right)\right),(x,(u, \alpha),(v, \zeta)) \in(\bar{x}(t), \bar{u}(t), \bar{\alpha}(t), \bar{v}(t), \bar{\zeta}(t))+$ $\varepsilon \bar{B}$ and for almost all $t \in[a, b]$,

$$
\begin{aligned}
& \mid \begin{array}{l}
\nabla_{x,(u, \alpha),(v, \zeta)} \psi\left(t, x^{\prime},\left(u^{\prime}, \alpha^{\prime}\right),\left(v^{\prime}, \zeta^{\prime}\right)\right)-\nabla_{x,(u, \alpha),(v, \zeta)} \psi(t, x,(u, \alpha),(v, \zeta)) \mid \\
=\mid\left(\nabla_{x}[b, g](t, x, u, v)-\nabla_{x}[b, g]\left(t, x^{\prime}, u^{\prime}, v^{\prime}\right), \nabla_{u}[b, g](t, x, u, v)-\nabla_{u}[b, g]\left(t, x^{\prime}, u^{\prime}, v^{\prime}\right), 0,\right. \\
\left.\nabla_{v}[b, g](t, x, u, v)-\nabla_{v}[b, g]\left(t, x^{\prime}, u^{\prime}, v^{\prime}\right), 0\right) \mid \\
\leq \tilde{\theta}\left(\left|\left(x^{\prime}, u^{\prime}, v^{\prime}\right)-(x, u, v)\right|\right) \leq \tilde{\theta}\left(\left|\left(x^{\prime},\left(u^{\prime}, \alpha^{\prime}\right),\left(v^{\prime}, \zeta^{\prime}\right)\right)-(x,(u, \alpha),(v, \zeta))\right|\right) .
\end{array}
\end{aligned}
$$

It remains to prove the uniform bound in (H4). Note that, for almost all $t \in[a, b]$,

$$
\begin{array}{r}
\left|\nabla_{x} \psi(t, \bar{x}(t), \bar{u}(t), \bar{\alpha}(t), \bar{v}(t), \bar{\zeta}(t))\right|+\left|\nabla_{u, \alpha} \psi(t, \bar{x}(t), \bar{u}(t), \bar{\alpha}(t), \bar{v}(t), \bar{\zeta}(t))\right| \\
+\left|\nabla_{v, \zeta} \psi(t, \bar{x}(t), \bar{u}(t), \bar{\alpha}(t), \bar{v}(t), \bar{\zeta}(t))\right| \\
\leq\left|\nabla_{x}[b, g](t, \bar{x}(t), \bar{u}(t), \bar{v}(t))\right|+\left|\nabla_{u}[b, g](t, \bar{x}(t), \bar{u}(t), \bar{v}(t))\right|+|E(t)| \\
+\left|\nabla_{v}[b, g] g(t, \bar{x}(t), \bar{u}(t), \bar{v}(t))\right|+|Z(t)| \leq K_{\psi},
\end{array}
$$

where the existence of $K_{\psi}>0$ is due to the fact that $|Z(t)|=1, t \mapsto g(t, \bar{x}(t), \bar{u}(t))$ is uniformly bounded by assumption which yields uniform boundedness of $E(t)$. This completes the proof of (H4).

Step 3: Finally we apply Theorem 3.1 to prove (i)-(iv).

By Step 2, Theorem 3.1 is applicable to $\left(P_{\text {aux }}\right)$ and therefore there exist $p \in W^{1,1}\left([a, b] ; \mathbb{R}^{n}\right)$, $\left(\eta^{1}, \eta^{2}\right) \in L^{1}\left([a, b] ; \mathbb{R}^{k_{v}} \times \mathbb{R}^{m_{g}}\right)$ and $\lambda \geq 0$ such that, for almost all $t \in[a, b]$,

$$
\begin{aligned}
\left(-\dot{p}(t), 0, \eta^{1}(t), 0, \eta^{2}(t)\right) & \in \operatorname{co~} \partial_{x, u, v, \alpha, \zeta} H(t, \bar{x}(t), p(t), q(t), r(t), \bar{u}(t), \bar{v}(t), \bar{\alpha}(t), \bar{\zeta}(t)) \\
\left(\eta^{1}(t), \eta^{2}(t)\right) & \in \operatorname{co~} N_{V(t)}(\bar{v}(t)) \times \operatorname{co~} N_{\mathcal{Z}}(\bar{\zeta}(t)) \\
(p(a),-p(b)) & \in N_{C}(\bar{x}(a), \bar{x}(b))+\lambda \partial l(\bar{x}(a), \bar{x}(b))
\end{aligned}
$$

where $\mathcal{Z}=\mathbb{R}_{\geq 0}^{m_{g}}$ and

$H(t, x, p, q, r, u, v, \alpha, \zeta)=\langle p, f(t, x, u, v)\rangle+\langle q, b(t, x, u, v)\rangle+\langle r, g(t, x, u, v)+E(t) \alpha(t)+Z(t) \zeta(t)\rangle$.

Observe that $N_{\mathcal{Z}}(\bar{\zeta}(t))=\mathbb{R}_{\leq 0}^{m_{g}}$. Since $\bar{\zeta}_{i}(t)=-g_{i}(t, \bar{x}(t), \bar{u}(t))=0$ if $i \in \mathcal{I}_{a}(t)$, and $\bar{\zeta}_{i}(t)>0$ if $i \in \mathcal{I}_{c}(t)$, we deduce from (4.5) and (4.6) that

$$
r_{i}(t)=0, \text { if } \quad g_{i}(t, \bar{x}(t), \bar{u}(t), \bar{v}(t))<0, \quad \text { and } \quad r_{i}(t) \leq 0, \text { if } \quad g_{i}(t, \bar{x}(t), \bar{u}(t), \bar{v}(t))=0 .
$$

Hence $\lambda, \eta=\eta_{1}, p, q$ and $r$ satisfy (ii)-(v). Note that $\bar{\psi}_{u, \alpha}(t)$ and $(q(t), r(t))$ take the role of $\Upsilon(t)$ and $q(t)$ in Theorem 3.1, respectively. Now by Step 2 and (H4) there exists an $M>0$ such that (3.1) holds. Applying (3.2) yields (3.3). This completes the proof. 
Acknowledgments: The support to Maria do Rosário de Pinho by Fundação para a Ciência e Tecnologia, PBICT/CEG/2438/95, Portugal is greatly acknowledged as well as numerous discussions with Prof. Vera Zeidan. This work was done while Achim Ilchmann was on study leave at the Faculdade de Eng. Universidade do Porto; the hospitality of the Instituto de Sistemas e Robótica, and the support by Fundação para a Ciência e Tecnologia, Praxis XXI, Portugal (BCC/20279/99), are gratefully acknowledged.

\section{References}

[1] HESTENES M.R., Calculus of Variations and Optimal Control Theory, Wiley, New York, (1966).

[2] NEUSTADT L.W., Optimization, A Theory of Necessary Conditions, Princeton University Press, New Jersey, (1976).

[3] PALES Z. \& ZEIDAN V., First- and second order necessary conditions for control problems with constraints, Transc. American Mathematical Society, 346, 421-453 (1994).

[4] STEFANI G. \& ZEZZA P.L., Optimality conditions for constrained control problems, SIAM J. Control and Optim., 34, 635-659 (1996).

[5] DE PINHO M.D.R., On the weak maximum principle for optimal control for problems with state-dependent control constraints, Nonlinear Analysis, Theo. Applic., 30, Proc. 2nd World Congress of Nonlinear Analysts, 2481-2488 (1997).

[6] DEVDARYANI E.N. \& LEDYAEV Y.S., Maximum principle for implicit control systems, Appl. Math. Optim., 40, 79-103 (1996).

[7] DE PINHO M.D.R., VINTER R.V. \& ZHENG H., A maximum principle for optimal control problems with mixed constraints, accepted for publication IMA J. Math. Control and Information, (2001).

[8] N. P. OSMOLOVSKII, Second order conditions for a weak local minimum in an optimal control problem (necessity and sufficiency), Soviet Math. Dokl., 16, 1480-1484, (1975).

[9] DE PINHO M.D.R. \& VINTER R.V., Necessary conditions for optimal control problems involving nonlinear differential algebraic equations, J. Math. Anal. and Applications, 212, 493-516 (1997).

[10] MORDUKHOVICH B.S., Maximum principle in problems of time optimal control with nonsmooth constraints, Journal of Appl. Math. Mech., 40, 960-969 (1976).

[11] MORDUKHOVICH B.S., Approximation Methods in Problems of Optimization and Control, Nakua, Moscow (1988); 2nd edition to be published by Wiley-Interscience. 
[12] ROCKAFELlAR R.T. \& WETS B., Variational Analysis, Springer, Berlin, (1998).

[13] CLARKE F.H., Optimization and Nonsmooth Analysis, Wiley, New York, (1983).

[14] DE PINHO M.D.R. \& VINTER R.V., An Euler Lagrange inclusion for optimal control problems, IEEE Trans. Aut. Control, AC-40, 1191-1198 (1995).

[15] HORN R.A. \& JOHNSON C.R. Matrix Analysis, Cambridge Press, (1985).

[16] RUDIN W., Principles of Mathematical Analysis, 3rd ed. McGraw-Hill, New York (1976). 